\title{
Second-order mean-field susceptible-infected-susceptible epidemic threshold
}

\author{
E. Cator ${ }^{*}$ and P. Van Mieghem ${ }^{\dagger}$ \\ Faculty of Electrical Engineering, Mathematics and Computer Science, P.O. Box 5031, 2600 GA Delft, The Netherlands
}

(Received 23 December 2011; published 14 May 2012)

\begin{abstract}
Given the adjacency matrix $A$ of a network, we present a second-order mean-field expansion that improves on the first-order $N$-intertwined susceptible-infected-susceptible (SIS) epidemic model. Unexpectedly, we found that, in contrast to first-order, second-order mean-field theory is not always possible: the network size $N$ should be large enough. Under the assumption of large $N$, we show that the crucial and characterizing quantity, the SIS epidemic threshold $\tau_{c}$, obeys an eigenvalue equation, more complex than the one in the first-order $N$-intertwined model. However, the resulting epidemic threshold is more accurate: $\tau_{c}^{(2)}=\tau_{c}^{(1)}+O\left(\frac{\tau_{c}^{(1)}}{N}\right)$, where the first-order epidemic threshold is $\tau_{c}^{(1)}=\frac{1}{\lambda_{1}(A)}$ and where $\lambda_{1}(A)$ is the spectral radius of the adjacency matrix $A$.
\end{abstract}

DOI: 10.1103/PhysRevE.85.056111

PACS number(s): 89.75.Hc, 89.20.-a

\section{SUSCEPTIBLE-INFECTED-SUSCEPTIBLE EPIDEMICS}

We consider a virus spread in an undirected graph $G(N, L)$ with $N$ nodes and $L$ links specified by a symmetric adjacency matrix $A$. The state of a node $i$ is specified by a Bernoulli random variable $X_{i} \in\{0,1\}: X_{i}=0$ for a healthy node and $X_{i}=1$ for an infected node. A node $i$ at time $t$ can be in one of two states: infected, with probability $v_{i}(t)=\operatorname{Pr}\left[X_{i}(t)=1\right]$ or healthy, with probability $1-v_{i}(t)$, but susceptible to the virus. We assume that the curing process per node $i$ is a Poisson process with rate $\delta$, and that the infection rate per link is a Poisson process with rate $\beta$. This is the general description of the simplest type of a susceptible-infectedsusceptible (SIS) virus spread model in a network, to which we confine ourselves in this article, although other variants such as susceptible-infected-recovered (SIR) exist [1]. This SIS model can be expressed exactly in terms of a continuous-time Markov model with $2^{N}$ states as shown in Ref. [2]. Unfortunately, the exponentially increasing state space with $N$ prevents the determination of the set of $\left\{v_{i}(t)\right\}_{1 \leqslant i \leqslant N}$ in realistically sized complex networks, which has triggered a spur of research to find good approximate solutions.

A remarkable property of the SIS model is the appearance of a phase transition $[3,4]$ when the effective infection rate $\tau=\frac{\beta}{\delta}$ approaches the epidemic threshold $\tau_{c}$. Below the epidemic threshold, $\tau<\tau_{c}$, the network is virus free in the steady state, while for $\tau>\tau_{c}$, there is a fraction of nodes that remains infected for an exponentially long time. The knowledge of the epidemic threshold $\tau_{c}$ for any graph is an important practical tool to predict and avoid infections in networks. Numerous immunization strategies [5,6] exist that modify (mostly increase) the epidemic threshold $\tau_{c}$. In spite of some asymptotic results [7], the determination of the epidemic threshold $\tau_{c}$ for an arbitrary graph is still a surprisingly difficult open problem. The simple SIS epidemics is considered as a first-order model for real virus spread, both in cyberspace as in the real world; the real epidemics can only be more complex. Hence, our currently inaccurate knowledge of the epidemic threshold $\tau_{c}$ in the simplest SIS model even gains in gravity when realizing that real biological virus epidemics

\footnotetext{
*e.a.cator@tudelft.nl

†p.f.a.vanmieghem@tudelft.nl
}

(like Sars, Mexican flu, etc.) are believed to be an imminent threat to the human population. The role of the network plays an increasingly crucial role due to the mobility and speed of encounters of possibly infected nodes. These arguments motivate our goal to determine the SIS epidemic threshold $\tau_{c}$ in an arbitrary graph, given its adjacency matrix $A$.

In computer science, Ganesh et al. [8] and Wang et al. [9] made progress on the simple SIS epidemics in networks. Later, the Wang et al. discrete-time model [10] was corrected, after which it was also noticed by the physics community [11]. Pastor-Satorras and Vespignani [12] proposed a solution to SIS epidemics on random networks, rather than on fixed networks. Hence, instead of starting from a given and total network description via the adjacency matrix $A$, the neighbor degree, in probabilistic sense, was the central network representative. Based on the Pastor-Satorras and Vespignani heterogeneous mean-field model (HMF) [12], a large amount of subsequent work on the epidemic threshold appeared [3].

Here, we approximate the SIS epidemics in any graph by subsequent order expansions. The first-order mean-field expansion that approximates $E\left[X_{i}\right]$ has been proposed earlier in Ref. [2] and was called the $N$-intertwined SIS epidemic model, which is briefly reviewed in Sec. II. The analytic tractability, extensions, and several insights on epidemics deduced from the $N$-intertwined SIS epidemic model are shown in Ref. [13]. In particular, the first-order epidemic threshold equals $\tau_{c}^{(1)}=\frac{1}{\lambda_{1}(A)} \leqslant \tau_{c}$, where $\lambda_{1}(A)$ is the largest eigenvalue of the adjacency matrix. Section III presents the second-order mean-field expansion that approximates the joint moments $E\left[X_{i} X_{j}\right]=\operatorname{Pr}\left[X_{i}=1, X_{j}=1\right]$ between any two nodes $i$ and $j$ in the graph $G$. The major insights that we can draw from our study are twofold: (a) Although in most cases, increasingly higher-order expansions are more accurate, these higher-order expansions are not always physically meaningful and, hence, sometimes impossible. (b) When possible though, we show that the second-order approximation of the epidemic threshold $\tau_{c}^{(2)}$ is again equal to the inverse of the largest eigenvalue of some matrix $H$, specified in Eq. (8), and that $\tau_{c}^{(2)}=\tau_{c}^{(1)}+O\left(\frac{\tau_{c}^{(1)}}{N}\right)$. This result is in line with expectations: a second-order approximation returns a more accurate epidemic threshold $\tau_{c}^{(2)}$ than the first-order $\tau_{c}^{(1)}$. The drawback of the second-order mean-field expansion is that the relation of the matrix $H$ to the underlying network is far less intuitive than 
for the adjacency matrix $A$, that is prominent in the first-order mean-field expansion.

\section{FIRST-ORDER MEAN-FIELD APPROXIMATION: THE $N$-INTERTWINED SUSCEPTIBLE-INFECTED-SUSCEPTIBLE EPIDEMIC MODEL}

In Ref. [2], a mean-field approximation is presented that replaces the random variable $\sum_{j=1}^{N} a_{i j} X_{j}(t)$ of the number of infected neighbors of node $i$ by its expectation $\sum_{j=1}^{N} a_{i j} v_{j}(t)$, leading to

$$
\frac{d v_{i}(t)}{d t}=\beta\left[1-v_{i}(t)\right] \sum_{j=1}^{N} a_{i j} v_{j}(t)-\delta v_{i}(t) .
$$

The governing differential Eq. (1) in the $N$-intertwined model for each node $i \in G$ has the following physical interpretation: the time derivative of the infection probability of a node $i$ consists of two competing processes: (i) while healthy with probability $\left[1-v_{i}(t)\right]$, all infected neighbors, of which there are expected to be $\sum_{j=1}^{N} a_{i j} v_{j}(t)$, try to infect the node $i$ with rate $\beta$ and (ii) while infected with probability $v_{i}(t)$, the node $i$ is cured at rate $\delta$.

Writing the set of Eqs. (1) for all nodes in matrix form with the vector

$$
v(t)=\left[v_{1}(t) v_{2}(t) \cdots v_{N}(t)\right]^{T}
$$

we arrive at

$$
\frac{d v(t)}{d t}=\operatorname{diag}\left[1-v_{i}(t)\right] \beta A v(t)-\delta v,
$$

where $\operatorname{diag}\left[v_{i}(t)\right]$ is the diagonal matrix with elements $v_{1}(t), v_{2}(t), \ldots, v_{N}(t)$.

Omitting the time dependence, the governing Eq. (1) is rewritten in terms of expectations with $v_{i}=\operatorname{Pr}\left[X_{i}=1\right]=$ $E\left[X_{i}\right]$ as

$$
\frac{d E\left[X_{i}\right]}{d t}=E\left[-\delta X_{i}+\beta\left(1-X_{i}\right) \sum_{j=1}^{N} a_{i j} E\left[X_{j}\right]\right],
$$

which should be compared with the exact companion in Eq. (5) below. The first-order approximation consists of replacing $E\left[X_{i} X_{j}\right]$

$$
\begin{aligned}
E\left[X_{i} X_{j}\right] & =\operatorname{Pr}\left[X_{i}=1, X_{j}=1\right] \\
& =\operatorname{Pr}\left[X_{j}=1 \mid X_{i}=1\right] \operatorname{Pr}\left[X_{i}=1\right]
\end{aligned}
$$

by $E\left[X_{i}\right] E\left[X_{j}\right]=\operatorname{Pr}\left[X_{i}=1\right] \operatorname{Pr}\left[X_{j}=1\right]$. Because a given infection at node $i$ cannot cure another node $j$, but can only positively influence the probability of infection at node $j$, it holds that $\operatorname{Pr}\left[X_{j}=1 \mid X_{i}=1\right] \geqslant \operatorname{Pr}\left[X_{j}=1\right]$ and $E\left[X_{i} X_{j}\right] \geqslant E\left[X_{i}\right] E\left[X_{j}\right]$. Hence, the replacement of $E\left[X_{i} X_{j}\right]$ by $E\left[X_{i}\right] E\left[X_{j}\right]$ results in a more positive right-hand side in Eq. (3). Consequently as reported in Ref. [2], the first order approximation in the $N$-intertwined SIS model upperbounds the exact probability $v_{i}(t)$ of infection.

\section{SECOND-ORDER MEAN-FIELD APPROXIMATION AND JOINT EXPECTATIONS $E\left[X_{i} X_{j}\right]$}

In this section, we consider the joint expectation $E\left[X_{i} X_{j}\right]$ that satisfies for $i \neq j$,

$$
\begin{aligned}
\frac{d E\left[X_{i} X_{j}\right]}{d t} & =E\left[-2 \delta X_{i} X_{j}+\beta X_{j}\left(1-X_{i}\right) \sum_{k=1}^{N} a_{i k} X_{k}+\beta X_{i}\left(1-X_{j}\right) \sum_{k=1}^{N} a_{j k} X_{k}\right] \\
& =-2 \delta E\left[X_{i} X_{j}\right]+\beta \sum_{k=1}^{N} a_{i k} E\left[X_{j} X_{k}\right]+\beta \sum_{k=1}^{N} a_{j k} E\left[X_{i} X_{k}\right]-\beta \sum_{k=1}^{N}\left(a_{i k}+a_{j k}\right) E\left[X_{i} X_{j} X_{k}\right]
\end{aligned}
$$

and when $i=j$,

$$
\frac{d E\left[X_{i}\right]}{d t}=E\left[-\delta X_{i}+\beta\left(1-X_{i}\right) \sum_{j=1}^{N} a_{i j} X_{j}\right] .
$$

Indeed, in a time-dependent Markov process, we can ignore the occurrence of multiple events in the arbitrarily small time interval $[t, t+d t]$. The right-hand side of the first equation states that the change in the joint expectation is due to a curing or an infection in either node $i$ or node $j$ (not in both due to the Markov property). Thus, curing of node $i$ contributes to the change in the product $X_{i} X_{j}$ by $\left(-\delta X_{i}\right) X_{j}$ and curing in node $j$ by $X_{i}\left(-\delta X_{j}\right)$. The infection contribution is similarly deduced.

The second-order mean-field approximation consists of replacing $\sum_{k=1 ; k \neq i, j}^{N} a_{j k} E\left[X_{i} X_{j} X_{k}\right]$ by $E\left[X_{i} X_{j}\right] \sum_{k=1 ; k \neq i, j}^{N}$ $a_{j k} E\left[X_{k}\right]$. We arrive at

$$
\frac{d E\left[X_{i} X_{j}\right]}{d t}=-2 \delta E\left[X_{i} X_{j}\right]+\beta \sum_{k=1}^{N} a_{i k} E\left[X_{j} X_{k}\right]+\beta \sum_{k=1}^{N} a_{j k} E\left[X_{i} X_{k}\right]-\beta E\left[X_{i} X_{j}\right] \sum_{k=1 ; k \neq i, j}^{N}\left(a_{i k}+a_{j k}\right) E\left[X_{k}\right]-2 \beta a_{i j} E\left[X_{i} X_{j}\right]
$$


or, rewritten as

$$
\begin{aligned}
\frac{d E\left[X_{i} X_{j}\right]}{d t}= & -2\left(\delta+\beta a_{i j}\right) E\left[X_{i} X_{j}\right]+\beta \sum_{k=1}^{N} a_{i k} E\left[X_{j} X_{k}\right]+\beta \sum_{k=1}^{N} a_{j k} E\left[X_{i} X_{k}\right]-\beta E\left[X_{i} X_{j}\right] \sum_{k=1}^{N} a_{i k} E\left[X_{k}\right] \\
& +\beta a_{i j} E\left[X_{i} X_{j}\right] E\left[X_{j}\right]-\beta E\left[X_{i} X_{j}\right] \sum_{k=1}^{N} a_{j k} E\left[X_{k}\right]+\beta a_{j i} E\left[X_{i} X_{j}\right] E\left[X_{i}\right] .
\end{aligned}
$$

When $i=j$, we do not need to make any approximation,

$$
\frac{d E\left[X_{i}\right]}{d t}=-\delta E\left[X_{i}\right]+\beta \sum_{j=1}^{N} a_{i j} E\left[X_{j}\right]-\beta \sum_{j=1}^{N} a_{i j} E\left[X_{i} X_{j}\right]
$$

We define the symmetric matrix $Z$ with elements $z_{i j}=z_{j i}=E\left[X_{i} X_{j}\right]$ when $i \neq j$, and $z_{i i}=E\left[X_{i}^{2}\right]=E\left[X_{i}\right]=v_{i}$. Again as in the first-order mean-field $N$-intertwined model, we define the vector $v$ such that $v_{i}=E\left[X_{i}\right]$. The governing set of differential equations is

$$
\begin{aligned}
& \frac{d z_{i j}}{d t}=-2\left(\delta+\beta a_{i j}\right) z_{i j}+\beta \sum_{k=1}^{N} a_{i k} z_{k j}+\beta \sum_{k=1}^{N} a_{j k} z_{k i}-\beta z_{i j} \sum_{k=1}^{N}\left(a_{i k}+a_{j k}\right) v_{k}+\beta a_{i j} z_{i j}\left(v_{i}+v_{j}\right) \quad \text { if } \quad i \neq j \\
& \frac{d z_{i i}}{d t}=\frac{d v_{i}}{d t}=-\delta v_{i}+\beta \sum_{k=1}^{N} a_{i k} v_{k}-\beta \sum_{k=1}^{N} a_{i k} z_{k i} \quad \text { if } \quad i=j .
\end{aligned}
$$

In the second-order approximation, we replace, for $i \neq j$, $i \neq k$, and $j \neq k$,

$$
\begin{aligned}
E\left[X_{i} X_{j} X_{k}\right] & =\operatorname{Pr}\left[X_{i}=1, X_{j}=1, X_{k}=1\right] \\
& =\operatorname{Pr}\left[X_{k}=1 \mid X_{i}=1, X_{j}=1\right] \operatorname{Pr}\left[X_{i}=1, X_{j}=1\right]
\end{aligned}
$$

by $E\left[X_{i} X_{j}\right] E\left[X_{k}\right]$. Since an infection at nodes $i$ and $j$ can only negatively affect the state of infection in node $k$ in the sense that node $k$ can only get infected (not cured), we have that

$$
\operatorname{Pr}\left[X_{k}=1 \mid X_{i}=1, X_{j}=1\right] \geqslant \operatorname{Pr}\left[X_{k}=1\right] .
$$

Hence, similarly as in the first-order approximation, the second-order approximation upper bounds the exact infection pair correlation $E\left[X_{i} X_{j}\right]$. Because the pair correlation $E\left[X_{i} X_{j}\right]$ is taken into account, the second-order approximation is more accurate than the first-order, that assumes independence.

Before proceeding, we observe that the above method is readily extended to a third-order approximation in $E\left[X_{i} X_{j} X_{k}\right]$, in which the fourth-order term $E\left[X_{i} X_{j} X_{k} X_{l}\right]$ is replaced by $E\left[X_{i} X_{j} X_{k}\right] E\left[X_{l}\right]$. Using the same arguments as above, that third-order expansion again upper bounds the exact SIS model. In conclusion, the method can be generalized to any order $m<N$ using the same type of replacement in the joint moments, which always leads to an upper bound. Subsequent order expansions are increasingly accurate, but the $m$ th order expansion involves $N^{m}$ unknowns to be solved. Somewhere, a tradeoff between computational complexity and accuracy will determine what order is appropriate. In future work, we plan to concentrate on $m=2$ and show what can be concluded about the steady state and the approximation $\tau_{c}^{(2)}$ of the epidemic threshold $\tau_{c}$.

\section{A. Fundamental limitation}

When $N \leqslant 5$ is small, however, numerical computations have shown that the resulting set of differential equations (6) can be unstable (in the sense that $z_{i j}=\operatorname{Pr}\left[X_{i}=1, X_{j}=1\right]$ can grow unboundedly, which is meaningless). The discovery of such anomalies in small networks is understandable, because in the exact SIS Markov model with $2^{N}$ states, there is no epidemic threshold and the steady state is the healthy network state (due to the absorbing state in the Markov chain). The observed instability of the set (6) is caused by the fact that given all $E\left[X_{i} X_{j}\right]$, there might not exist a distribution for which the replacement of $E\left[X_{i} X_{j} X_{k}\right]$ by $E\left[X_{i} X_{j}\right] E\left[X_{k}\right]$ is valid, while, in the first-order meanfield approximation, for all choices of $E\left[X_{i}\right]$, the independent distribution can be chosen such that $E\left[X_{i} X_{j}\right]=$ $E\left[X_{i}\right] E\left[X_{j}\right]$.

Let us consider in Eq. (4) the term, approximated in the second-order mean-field expansion,

$$
\begin{aligned}
& \sum_{k=1}^{N}\left(a_{i k}+a_{j k}\right) E\left[X_{i} X_{j} X_{k}\right] \\
& \quad=2 a_{i j} E\left[X_{i} X_{j}\right]+\sum_{k=1 ; k \neq i, j}^{N}\left(a_{i k}+a_{j k}\right) E\left[X_{i} X_{j} X_{k}\right]
\end{aligned}
$$

and denote $r_{i j k}=E\left[X_{i} X_{j} X_{k}\right]$. Since $X_{k}$ is a Bernoulli random variable,

$$
r_{i j k}=E\left[X_{i} X_{j} X_{k}\right]=\operatorname{Pr}\left[X_{i}=1, X_{j}=1, X_{k}=1\right]
$$

we can write the seven remaining possible combinations of zero and ones as a function of $r_{i j k}$. For example,

$\operatorname{Pr}\left[X_{i}=1, X_{j}=1, X_{k}=0\right]=E\left[X_{i} X_{j}\right]-r_{i j k}=z_{i j}-r_{i j k}$ 
and

$$
\begin{aligned}
& \operatorname{Pr}\left[X_{i}=1, X_{j}=0, X_{k}=1\right]=z_{i k}-r_{i j k} \\
& \operatorname{Pr}\left[X_{i}=0, X_{j}=1, X_{k}=1\right]=z_{j k}-r_{i j k},
\end{aligned}
$$

from which $r_{i j k} \leqslant \min \left(z_{i j}, z_{i k}, z_{j k}\right)$. Further, from

$$
\begin{aligned}
& \operatorname{Pr}\left[X_{i}=1, X_{j}=0, X_{k}=0\right]+\operatorname{Pr}\left[X_{i}=1, X_{j}=0, X_{k}=1\right] \\
& \quad=\operatorname{Pr}\left[X_{i}=1, X_{j}=0\right]=E\left[X_{i}\right]-z_{i j}
\end{aligned}
$$

it follows that

$$
\operatorname{Pr}\left[X_{i}=1, X_{j}=0, X_{k}=0\right]=v_{i}-z_{i j}-z_{i k}+r_{i j k} .
$$

Thus, since all probabilities must lie between 0 and 1 , a set of bounds for $r_{i j k}$ can be deduced. The argument shows that replacement of $r_{i j k}=E\left[X_{i} X_{j} X_{k}\right]$ by $E\left[X_{i} X_{j}\right] E\left[X_{k}\right]=z_{i j} v_{k}$ needs to be checked. For example, if $v_{k}$ is close to one and $z_{i j}>z_{i k}$ and $z_{i j}>z_{j k}$, then $r_{i j k}$ replaced by $z_{i j} v_{k}$ may violate the general bound $r_{i j k} \leqslant \min \left(z_{i j}, z_{i k}, z_{j k}\right)$ for three Bernoulli random variables. There does not seem to be an easy way to incorporate these probabilistic limitations into the set of differential equations. It turns out that the second-order mean-field approximation is valid for sufficiently large $N$ and we will illustrate possible unrealistic consequences for smaller $N$.

\section{B. The steady state}

The steady state is characterized by $\frac{d z_{i j}}{d t}=0$ and $\lim _{t \rightarrow \infty} z_{i j}=z_{i j \infty}$, that obeys the set of nonlinear equations (defining $\tau=\beta / \delta$ )

$$
\begin{aligned}
& 0=-\left(1+\tau a_{i j}\right) z_{i j \infty}+\frac{\tau}{2} \sum_{k=1}^{N} a_{i k} z_{k j \infty}+\frac{\tau}{2} \sum_{k=1}^{N} a_{j k} z_{k i \infty}-\frac{\tau}{2} z_{i j \infty} \sum_{k=1}^{N}\left(a_{i k}+a_{j k}\right) v_{k \infty}+\frac{\tau}{2} a_{i j} z_{i j \infty}\left(v_{i \infty}+v_{j \infty}\right) \quad \text { if } \quad i \neq j \\
& 0=-v_{i \infty}+\tau \sum_{k=1}^{N} a_{i k} v_{k \infty}-\tau \sum_{k=1}^{N} a_{i k} z_{k i \infty} \quad \text { if } \quad i=j .
\end{aligned}
$$

The corresponding matrix form, valid for the off-line diagonal elements, is

$$
0=-(I+\tau A) \bullet Z_{\infty}+\frac{\tau}{2}\left(A Z_{\infty}+Z_{\infty} A\right)-\frac{\tau}{2}\left\{\operatorname{diag}\left[\left(A v_{\infty}\right)_{i}\right] Z_{\infty}+Z_{\infty} \operatorname{diag}\left[\left(A v_{\infty}\right)_{i}\right]-A \bullet\left[Z_{\infty} \operatorname{diag}\left(v_{\infty i}\right)+\operatorname{diag}\left(v_{\infty i}\right) Z_{\infty}\right]\right\}
$$

where $(R \bullet S)_{i j}=r_{i j} s_{i j}$. The diagonal elements obey the vector equation (defining $u$ as the vector of all ones)

$$
-v_{\infty}+\tau A v_{\infty}-\tau \operatorname{diag}\left[\left(A Z_{\infty}\right)_{i i}\right] u=0 .
$$

In order to obtain a matrix equation that is valid for all entries, we need to correct the diagonal elements and arrive at

$$
\begin{aligned}
0= & -(I+\tau A) \bullet Z_{\infty}+\frac{\tau}{2}\left(A Z_{\infty}+Z_{\infty} A\right) \\
& -\frac{\tau}{2}\left\{\operatorname{diag}\left[\left(A v_{\infty}\right)_{i}\right] Z_{\infty}+Z_{\infty} \operatorname{diag}\left[\left(A v_{\infty}\right)_{i}\right]\right. \\
& \left.-A \bullet\left[Z_{\infty} \operatorname{diag}\left(v_{\infty i}\right)+\operatorname{diag}\left(v_{\infty i}\right) Z_{\infty}\right]\right\} \\
& +\tau \operatorname{diag}\left(\left(A v_{\infty}\right)_{i}-2\left(A Z_{\infty}\right)_{i i}+\left\{\operatorname{diag}\left[\left(A v_{\infty}\right)_{i}\right] v_{\infty}\right\}_{i}\right) .
\end{aligned}
$$

Just as in the first-order mean-field approximation [2], the trivial solution is $Z_{\infty}=0$ and $v_{\infty}=0$. The epidemic threshold $\tau_{c}$ specifies the value of $\tau$ at which the first nonzero solution for elements of $Z_{\infty}$ appear. In order to find this critical value of $\tau$, we consider the solution of the above steady-state equation with very small norm. This means that the second-order terms (i.e., products of elements of $Z_{\infty}$ and $v_{\infty}$ ) become negligible, and we end up with

$$
\begin{aligned}
0= & -Z_{\infty}-\tau A \bullet Z_{\infty}+\frac{\tau}{2}\left(A Z_{\infty}+Z_{\infty} A\right) \\
& +\tau \operatorname{diag}\left[\left(A v_{\infty}\right)_{i}-2\left(A Z_{\infty}\right)_{i i}\right] .
\end{aligned}
$$

We proceed by vectorizing this matrix equation and define $\operatorname{vec}\left(Z_{\infty}\right)$, the $\left(N^{2} \times 1\right)$ vector consisting of all elements of $Z$ columnwise (see Ref. [14], p. 254),

$$
\begin{aligned}
\operatorname{vec}\left(Z_{\infty}\right)= & \left(z_{11}, z_{21}, \ldots, z_{N 1}, z_{12}, z_{22}, \ldots,\right. \\
& \left.z_{N 2}, \ldots, z_{1 N}, z_{2 N}, \ldots, z_{N N}\right)
\end{aligned}
$$

We define the $N^{2} \times N^{2}$ diagonal matrix $M$ by $m_{j j}=$ $1_{\{j=(i-1) N+i\}}$ for $1 \leqslant i \leqslant N$, where $1_{x}$ is the indicator function, so that $M \operatorname{vec}(Z)=\left(z_{11}, 0, \ldots, 0, z_{22}, 0, \ldots, z_{33}, 0, \ldots\right)$. Then, using the Kronecker product $\otimes$ and $J=u \cdot u^{T}$ is the all-one matrix, we have

$$
\begin{aligned}
0= & -\operatorname{vec}\left(Z_{\infty}\right)-\tau\{\operatorname{diag}[\operatorname{vec}(A)]\} \operatorname{vec}\left(Z_{\infty}\right) \\
& +\frac{\tau}{2}\left\{I_{N} \otimes A+A \otimes I_{N}\right\} \operatorname{vec}\left(Z_{\infty}\right) \\
& +\tau M(A \otimes J) M \operatorname{vec}\left(Z_{\infty}\right)-2 \tau M\left(A \otimes I_{N}\right) \operatorname{vec}\left(Z_{\infty}\right),
\end{aligned}
$$

which is written as an eigenvalue equation

$$
H \operatorname{vec}\left(Z_{\infty}\right)=\frac{1}{\tau} \operatorname{vec}\left(Z_{\infty}\right)
$$

where the $N^{2} \times N^{2}$ matrix $H$ is

$$
\begin{aligned}
H= & \frac{1}{2}\left\{I_{N} \otimes A+A \otimes I_{N}\right\}-\operatorname{diag}[\operatorname{vec}(A)] \\
& +M\left\{(A \otimes J) M-2\left(A \otimes I_{N}\right)\right\}
\end{aligned}
$$

with zero diagonal elements.

The first nonzero solution of $Z_{\infty}$, satisfying all symmetry properties, occurs when $\operatorname{vec}\left(Z_{\infty}\right)$ is the eigenvector of $H$ belonging to the largest eigenvalue $\lambda_{1}(H)=\frac{1}{\tau_{1}}$ so that $\tau_{1}$ is, indeed, the smallest value of $\tau$ at which $Z_{\infty}$ contains nonzero elements. Hence, the epidemic threshold deduced from the second-order mean-field expansion obeys

$$
\tau_{c}^{(2)}=\frac{1}{\lambda_{1}(H)}
$$

and it remains to specify $\lambda_{1}(H)$ in terms of the eigenstructure of the adjacency matrix $A$ of the network. 


\section{Possible unrealistic solution}

We remark that $H$ contains negative elements and is not symmetric (because of the last matrix $M\left(A \otimes I_{N}\right)$ due to $\operatorname{diag}\left[\left(A Z_{\infty}\right)_{i i}\right]$ that only selects from $A Z_{\infty}$ the diagonal elements via $M)$. For example, we see that

$$
\left\{I_{N} \otimes A+A \otimes I_{N}\right\}=\left\{I_{N} \otimes A+A \otimes I_{N}\right\}^{T}
$$

and

$$
\begin{aligned}
{[M(A \otimes J) M]^{T} } & =M^{T}(A \otimes J)^{T} M^{T} \\
& =M\left(A^{T} \otimes J^{T}\right) M=M(A \otimes J) M
\end{aligned}
$$

are symmetric. Moreover,

$$
\begin{aligned}
M\left(A \otimes I_{N}\right) \operatorname{vec}\left(Z_{\infty}\right) & =M(I \otimes A) \operatorname{vec}\left(Z_{\infty}\right) \\
& =\frac{1}{2} M\left\{I_{N} \otimes A+A \otimes I_{N}\right\} \operatorname{vec}\left(Z_{\infty}\right)
\end{aligned}
$$

illustrating that there are different expressions of $H$ but the matrix

$$
\begin{aligned}
& \left(M\left\{I_{N} \otimes A+A \otimes I_{N}\right\}\right)^{T} \\
& \quad=\left\{I_{N} \otimes A+A \otimes I_{N}\right\} . M \neq M .\left\{I_{N} \otimes A+A \otimes I_{N}\right\}
\end{aligned}
$$

is not symmetric.

It is well known that, if a matrix $H$ is not symmetric, its eigenvalues and eigenvector can be complex. Moreover, $H$ is not non-negative, which means that the eigenvector components belonging to $\lambda_{1}(H)$ are not necessarily positive (at least, the Perron-Frobenius theorem [14], that guarantees nonnegative eigenvector components, is not applicable). Finally, even when the eigenvector $w_{1}$ of $H$ belonging to $\lambda_{1}(H)$ has non-negative components, it represents $\operatorname{vec}\left(Z_{\infty}\right)$, in which $Z_{\infty}=Z_{\infty}^{T}$ is symmetric (and very likely positive definite). Hence, $w_{1}$ should possess additional symmetry properties. In particular for small $N$, such unrealistic solutions for $\operatorname{vec}\left(Z_{\infty}\right)$ and $\lambda_{1}(H)$ can occur. However, when $N$ is sufficiently large, their occurrence (based on some numerical checks) rapidly seems to decrease. Unfortunately, we cannot indicate for which $N$ these finite size effects disappear.

Assuming that $N$ is large enough, we can continue as follows.

\section{The epidemic threshold $\tau_{c}^{(2)}$}

We know ([14], p. 254) that the largest eigenvalue of the non-negative matrix $\frac{1}{2}\left\{I_{N} \otimes A+A \otimes I_{N}\right\}$ equals $\lambda_{1}(A)$, which leads to the first-order estimate of the epidemic threshold $\tau_{c}^{(1)}=\frac{1}{\lambda_{1}(A)}$. The corresponding eigenvector is $y_{1}=$ $x_{1} \otimes x_{1}$, where $x_{1}$ is the eigenvector of $A$ belonging to $\lambda_{1}(A)$. The matrix

$$
R=M^{T}(A \otimes J) M-2 M\left(A \otimes I_{N}\right)-\operatorname{diag}[\operatorname{vec}(A)]
$$

is not symmetric, relatively sparse, and can be regarded as a second-order correction to the first-order approximation in the $N$-intertwined model. The sums of the elements in $R$,

$$
\begin{aligned}
u^{T} M^{T}(A \otimes J) M u & =2 L=u^{T} A u \\
u^{T} M\left[\left(A \otimes I_{N}\right)\right] u & =2 L \\
u^{T} \operatorname{diag}[\operatorname{vec}(A)] u & =2 L
\end{aligned}
$$

imply that $u^{T} R u=-4 L$, while $u^{T}\left(\frac{1}{2}\left\{I_{N} \otimes A+A \otimes I_{N}\right\}\right)$ $u=2 L N$. Hence, the Rayleigh principle [14] shows that

$$
\begin{aligned}
\lambda_{1}(H) & \geqslant \frac{u^{T} H u}{u^{T} u}=\frac{2 L N-4 L}{N^{2}} \\
& =\frac{2 L}{N}\left(1-\frac{2}{N}\right)=E[D]\left(1-\frac{2}{N}\right),
\end{aligned}
$$

where $E[D]$ is the average degree of the network. The correction matrix $R$ is expected to have the effect to lower the largest eigenvalue so that $\lambda_{1}(H)<\lambda_{1}(A)$. We will elaborate this important observation in the remainder of this section.

Let us apply the Rayleigh principle, taking into account that $x_{1}^{T} x_{1}=\sum_{k=1}^{N}\left(x_{1}\right)_{k}^{2}=1$ and so $y_{1}^{T} y_{1}=1$,

$$
\lambda_{1}(H) \geqslant y_{1}^{T} H y_{1}=\lambda_{1}(A)+y_{1}^{T} R y_{1}
$$

where

$$
\begin{aligned}
y_{1}^{T} R y_{1}= & \left(x_{1}^{T} \otimes x_{1}^{T}\right) M(A \otimes J) M\left(x_{1} \otimes x_{1}\right) \\
& -2\left(x_{1}^{T} \otimes x_{1}^{T}\right) M\left(A \otimes I_{N}\right)\left(x_{1} \otimes x_{1}\right) \\
& -\left(x_{1}^{T} \otimes x_{1}^{T}\right) \operatorname{diag}[\operatorname{vec}(A)]\left(x_{1} \otimes x_{1}\right) \\
= & \sum_{m=1}^{N} \sum_{k=1}^{N}\left(x_{1}\right)_{m}^{2} a_{m k}\left(x_{1}\right)_{k}^{2}-2\left(x_{1}^{T} \otimes x_{1}^{T}\right) M \\
& \times\left(A x_{1} \otimes I_{N} x_{1}\right)-\sum_{m=1}^{N} \sum_{k=1}^{N}\left(x_{1}\right)_{m}^{2} a_{m k}\left(x_{1}\right)_{k}^{2} \\
= & -2 \lambda_{1}(A)\left(x_{1}^{T} \otimes x_{1}^{T}\right) M\left(x_{1} \otimes x_{1}\right) \\
= & -2 \lambda_{1}(A) \sum_{k=1}^{N}\left(x_{1}\right)_{k}^{4} .
\end{aligned}
$$

Hence,

$$
\lambda_{1}(H) \geqslant \lambda_{1}(A)\left(1-2 \sum_{k=1}^{N}\left(x_{1}\right)_{k}^{4}\right) .
$$

Further, using $\quad \sum_{k=1}^{N}\left(x_{1}\right)_{k}^{4} \leqslant \max _{1 \leqslant k \leqslant N}\left(x_{1}\right)_{k}^{2} \sum_{k=1}^{N}\left(x_{1}\right)_{k}^{2}=$ $\max _{1 \leqslant k \leqslant N}\left(x_{1}\right)_{k}^{2}$ and the Cauchy-Schwarz inequality, we find the bounds

$$
\frac{1}{N} \leqslant \sum_{k=1}^{N}\left(x_{1}\right)_{k}^{4} \leqslant \max _{1 \leqslant k \leqslant N}\left(x_{1}\right)_{k}^{2}<1 .
$$

In conclusion, the lower bound of $\lambda_{1}(H)$ is at most $\lambda_{1}(A)$ $\left(1-\frac{2}{N}\right)$.

Let $w_{1}$ be the normalized eigenvector of $H$ belonging to the largest eigenvalue $\lambda_{1}(H)$. The vector $w_{1}$ must all have nonnegative components because they reflect values of the joint moments $E\left[X_{i} X_{j}\right] \geqslant 0$. Again, application of the Rayleigh principle shows that

$$
\lambda_{1}(H) \leqslant \lambda_{1}(A)+w_{1}^{T} R w_{1} .
$$

As shown above, the sum of the elements of $R, u^{T} R u=-4 L$, is negative and $w_{1}^{T} R w_{1}$ is a weighted sum of $R$ 's elements, which, we argue, is negative. The matrix $R_{1}=M^{T}(A \otimes J) M$ contains precisely the same nonzero elements, equal to the adjacency elements $a_{i j}$, as $R_{2}=M\left[\left(A \otimes I_{N}\right)\right]$, though on 
different positions, so that

$$
\begin{aligned}
w_{1}^{T} & R_{1} w_{1}-2 w_{1}^{T} R_{2} w_{1} \\
= & \sum_{m=1}^{N} \sum_{k=1}^{N}\left[\left(R_{1}\right)_{m k}-2\left(R_{2}\right)_{m k}\right]\left(w_{1}\right)_{m}\left(w_{1}\right)_{k}
\end{aligned}
$$

is likely negative (due to the factor 2). In addition, since $w_{1}^{T} \operatorname{diag}[\operatorname{vec}(A)] w_{1}>0$, the quadratic form

$$
w_{1}^{T} R w_{1}=w_{1}^{T} R_{1} w_{1}-2 w_{1}^{T} R_{2} w_{1}-w_{1}^{T} \operatorname{diag}[\operatorname{vec}(A)] w_{1}
$$

is negative with overwhelming probability for sufficiently large $N$.

In conclusion, for sufficiently large $N$,

$$
\lambda_{1}(A)\left(1-2 \sum_{k=1}^{N}\left(x_{1}\right)_{k}^{4}\right) \leqslant \lambda_{1}(H) \leqslant \lambda_{1}(A)-\left|w_{1}^{T} R w_{1}\right|
$$

and these bounds, together with $\lambda_{1}(H) \geqslant E[D]\left(1-\frac{2}{N}\right)$, suggest that $\lambda_{1}(H) \simeq\left(1-\frac{2}{N}\right) \lambda_{1}(A) \simeq \lambda_{1}(A)-O\left(\frac{\lambda_{1}(A)}{N}\right)$. Equivalently, the epidemic threshold in the second-order approximation shifts to slightly higher values, thus $\tau_{c}^{(2)} \simeq \tau_{c}^{(1)}+$ $O\left(\frac{1}{N} \tau_{c}^{(1)}\right) \leqslant \tau_{c}$. The latter bound follows from the general consideration that subsequent higher-order mean-field expansions upper bound the real infection probability.

\section{Examples}

The epidemic threshold $\tau_{c}^{(2)}=\frac{1}{\lambda_{1}(H)}$ has been numerically computed, ignoring the possible imaginary part of the eigenvalues of the $N^{2} \times N^{2}$ matrix $H$ in Eq. (8). For large $N$, $\operatorname{Im}\left[\lambda_{1}(H)\right]$ is much smaller than $\operatorname{Re}\left[\lambda_{1}(H)\right]$, which justifies the neglect of $\operatorname{Im} \lambda_{1}(H)$. All numerical examples confirm that $\tau_{c}^{(2)}=\frac{1}{\lambda_{1}(H)}>\tau_{c}^{(1)}=\frac{1}{\lambda_{1}(A)}$ as made plausible in Sec. III B2.

For the complete graph $K_{N}$, we found that $\operatorname{Re}\left[\lambda_{1}(H)\right]=$ $N-2$, whereas $\lambda_{1}(A)=N-1$, so that $\tau_{c}^{(2)}=\frac{1}{N-2}>\tau_{c}^{(1)}=$ $\frac{1}{N-1}$. For a star, we found approximately that $\left\{\operatorname{Re}\left[\lambda_{1}(H)\right]\right\}^{2} \simeq$ $0.53 N-1.3$, whereas $\lambda_{1}^{2}(A)=N-1$, implying that $\tau_{c}^{(2)}$ is significantly larger than $\tau_{c}^{(1)}$. Figure 1 illustrates that $\tau_{c}^{(2)}=$ $\frac{1}{\lambda_{1}(H)}>\tau_{c}^{(1)}=\frac{1}{\lambda_{1}(A)}$ for the line topology or path with $N-$ 1 hops, which has the smallest largest adjacency eigenvalue among all connected graphs ([14], p. 125) with $N$ nodes, thus suggesting (and in line with physical expectation) the highest possible epidemic threshold $\tau_{c}$.

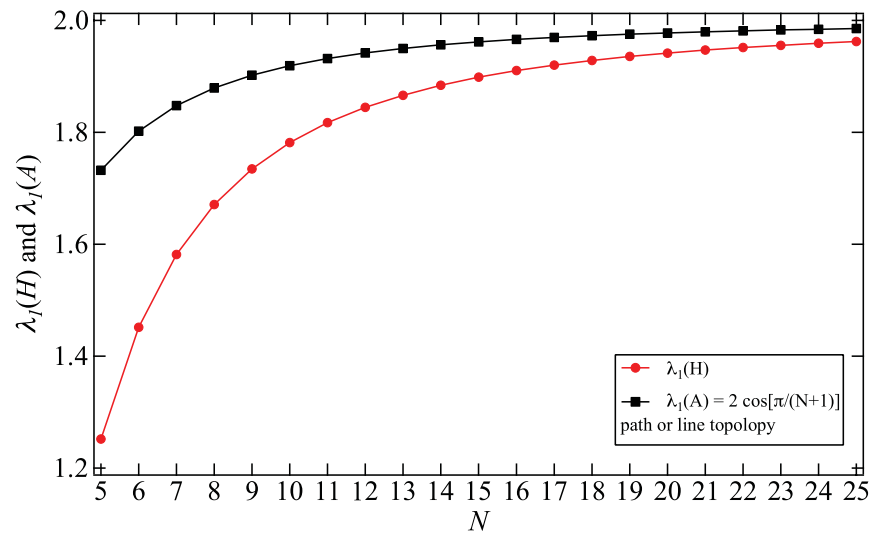

FIG. 1. (Color online) The largest eigenvalues of $A$ and $H$ for the line topology on $N$ nodes.

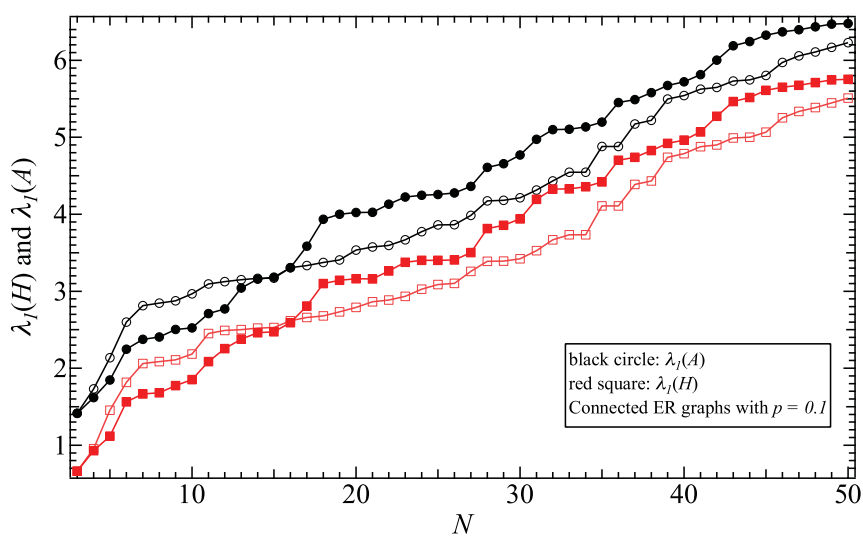

FIG. 2. (Color online) The largest eigenvalues of $A$ and $H$ for two realizations of the ER graph with link density $p=0.1$ (conditioned to be connected) versus $N$ nodes.

Figure 2 shows the largest eigenvalues of $A$ and $H$ in two realizations of connected Erdős-Rényi (ER) graphs $G_{p}(N)$ with link density $p=0.1$. The growth of $G_{p}(N)$ with $N$ is constructed so that each lower-sized ER graph is a subgraph [i.e., $G_{0.1}\left(n_{1}\right) \subset G_{0.1}\left(n_{2}\right)$ when $n_{1}<n_{2}$ ]. The difference $\lambda_{1}(A)-\lambda_{1}(H)$ per realization (hollow versus filled markers in Fig. 2) is remarkably constant.

\section{CONCLUSION}

Given an arbitrary network with adjacency matrix $A$, an accurate determination of the epidemic threshold $\tau_{c}$ in the SIS model remains a challenging quest. We have shown a second-order mean-field expansion that improves the accuracy over a first-order mean-field approximation at the price of a substantially more complex, but still feasible computation. Moreover, our analysis provides the computation of the joint moment or pair correlation $E\left[X_{i} X_{j}\right]=\operatorname{Pr}\left[X_{i}=1, X_{j}=1\right]$ between any pair $i$ and $j$ of nodes in the network. Whereas the first-order $N$-intertwined model gives the mean fraction $y(\tau)=E\left[\sum_{j=1}^{N} X_{j}\right]$ of infected nodes, the proposed second-order mean-field expansion allows us to compute the corresponding variance of the fraction of infected nodes, $\operatorname{Var}\left[\sum_{j=1}^{N} X_{j}\right]$. Recently, Gleeson [15] proposed a pairwise approximation, which can be regarded as a second-order improvement of the Pastor-Satorras and Vespignani HMF [12]. Although Gleeson [15] addressed the same problem, our approach has the advantage to upper bound the epidemic pair correlation $E\left[X_{i} X_{j}\right]=\operatorname{Pr}\left[X_{i}=1, X_{j}=1\right]$ and to provide a closed form expression (9) for any graph.

High-order mean-field expansions are argued to provide tight upper bounds to the exact solution, resulting in a sequence of more accurate lower bounds for the epidemic threshold $\tau_{c} \geqslant$ $\cdots \geqslant \tau_{c}^{(m)} \geqslant \tau_{c}^{(m-1)} \geqslant \cdots \geqslant \tau_{c}^{(2)}=\frac{1}{\lambda_{1}(H)} \geqslant \tau_{c}^{(1)}=\frac{1}{\lambda_{1}(A)}$. As an inherent drawback, the solution of the corresponding eigenvalue equation, such as (7) for $m=2$, is not always guaranteed to be physically correct, but very likely when the network size $N$ is sufficiently large.

One may argue that high-order mean-field expansions may not be the most suitable device to determine the epidemic threshold $\tau_{c}$ accurately. Finding additional structure in the exact Markov infinitesimal generator given in Ref. [2] may 
present a viable alternative. Another currently addressed idea is the removal of the troubling absorbing state in the SIS model by allowing, beside the neighbor infection, a constant, but very small self-infection rate at each node.

\section{ACKNOWLEDGMENTS}

This research was supported by EU FP7 Network of Excellence in Internet Science EINS (Project No. 288021).
[1] M. J. Keeling and K. T. D. Eames, J. R. Soc. Interface 2, 295 (2005).

[2] P. Van Mieghem, J. Omic, and R. E. Kooij, IEEE ACM T. Network. 17, 1 (2009).

[3] C. Castellano and R. Pastor-Satorras, Phys. Rev. Lett. 105, 218701 (2010).

[4] A. Barrat, M. Bartelemy, and A. Vespignani, Dynamical Processes on Complex Networks (Cambridge University Press, Cambridge, 2008).

[5] Y. Chen, G. Paul, S. Havlin, F. Liljeros, and H. E. Stanley, Phys. Rev. Lett. 101, 058701 (2008).

[6] P. Van Mieghem, D. Stevanović, F. A. Kuipers, C. Li, R. van de Bovenkamp, D. Liu, and H. Wang, Phys. Rev. E 84, 016101 (2011).

[7] R. Durrett, Proc. Nat. Acad. Sci. USA 107, 4491 (2010).
[8] A. Ganesh, L. Massoulié, and D. Towsley, IEEE INFOCOM, Miami, pp. 1455-1466, 2005.

[9] Y. Wang, D. Chakrabarti, C. Wang, and C. Faloutsos, in 22nd International Symposium on Reliable Distributed Systems (SRDS' 03) (IEEE Computer, Washington, DC, 2003), pp. 25-34.

[10] D. Chakrabarti, Y. Wang, C. Wang, J. Leskovec, and C. Faloutsos, ACM T. Inform. and Syst. 10, 1 (2008).

[11] S. Gómez, A. Arenas, J. Borge-Holthoefer, S. Meloni, and Y. Moreno, Europhys. Lett. 89, 38009 (2010).

[12] R. Pastor-Satorras and A. Vespignani, Phys. Rev. E 63, 066117 (2001).

[13] P. Van Mieghem, Computing 93, 147 (2011).

[14] P. Van Mieghem, Graph Spectra for Complex Networks (Cambridge University Press, Cambridge, 2011).

[15] J. P. Gleeson, Phys. Rev. Lett. 107, 068701 (2011). 